\title{
The Interactions Between Central Nervous System Cells and Topographically Modified Surfaces
}

\author{
Andrea M. P. Turner,* Natalie Dowell, ** Stephen W. P. Turner,* Ginger Withers, ${ }^{* * *}$ James \\ Turner, ${ }^{* *}$ Gary Banker, ${ }^{* * *}$ William Shain, ${ }^{* *}$ Harold G. Craighead* \\ * School of Applied \& Engineering Physics, Cornell University, Ithaca, NY 14853 \\ ** NYS DOH, Wadsworth Center, Albany, NY 12237 \\ *** Oregon Health \& Science University, Portland, OR 97201
}

Although scientists for the past 100 years have been studying the effects of surface contact on the growth and development of mammalian cells, it has only been during the last two decades that various researchers around the world have been employing the tools developed by the semiconductor industry to fabricate complex surface features for use in cell culture studies [1]. The work presented here concerns the study of the effects of structural cues on the attachment, spreading and outgrowth of mammalian central nervous system cells. This topic of research and others like it stand at the common boundaries between physics, engineering, and biology in the relatively new field of "Nanobiotechnology".

Our motivation for studying the interactions between central nervous system cells and topographically modified surfaces lies in the hypothesis that structural cues on the cellular size-scale might significantly impact the formation of detrimental glial scars around implanted neural devices. The formation of such an electrically insulating glial sheath renders a probe useless for long-term in vivo applications [2]. Thus, early work focused on the responses of astroglial cells to various topographically patterned substrates [3]. Focus was then redirected to study the effects of these same structural cues on the attachment and process outgrowth of hippocampal neurons as a function of the geometries presented to the cells.

Standard semiconductor fabrication techniques were used to fabricate micrometer-sized columnar structures in single-crystal silicon wafers (see Figure 1). Photolithography and reactive-ion etching were the cornerstone methods employed in the fabrication process. Methods of hot-embossing were also used to fabricate topographically patterned polymer substrates for use in time-lapse imaging. Substrates were patterned with a range of feature sizes from $0.5 \mu \mathrm{m}$ up to $2.0 \mu \mathrm{m}$, separated by a range of gaps from 0.5 to $5.0 \mu \mathrm{m}$.

Prior to cell culture, sterilized patterned substrates were incubated in $1 \mathrm{mg} / \mathrm{ml}$ solutions of poly-1lysine in borate buffer to promote neuron cell adhesion. Cells were then plated on surfaces and allowed to adhere and spread for periods from 24 hours to 21 days. At pre-determined time points, cells were fixed and stained immunochemically to visualize cell membranes, processes, and the expression of specific proteins via fluorescence microscopy (see Figure 2A). Fixed samples were also critical-point dried and gold-coated for scanning electron microscopy (SEM) analysis (see Figure 2B). Data contained in the fluorescence and SEM images were used to quantify the responses of hippocampal neurons to the columnar structures. The rates of process (axonal and dendritic) outgrowth and the degree of process alignment to the patterns on the substrate were measured as a function of the inter-pillar spacing, or geometric constraint, presented to the cell. Additionally, these results and the images obtained in time-lapse studies were used to assist in the development of 
heuristic models of neurite outgrowth on pillar surfaces. These results and their implications will be presented.

References:

[1] H.G. Craighead et al., Curr. Opin. Sol. St. Mater. Sci. 5 (2001) 177.

[2] J.N. Turner et al., Expt. Neuro. 156 (1999) 33.

[3] A.M.P. Turner et al., J. Biomed. Mater. Res. 51 (2000) 430.

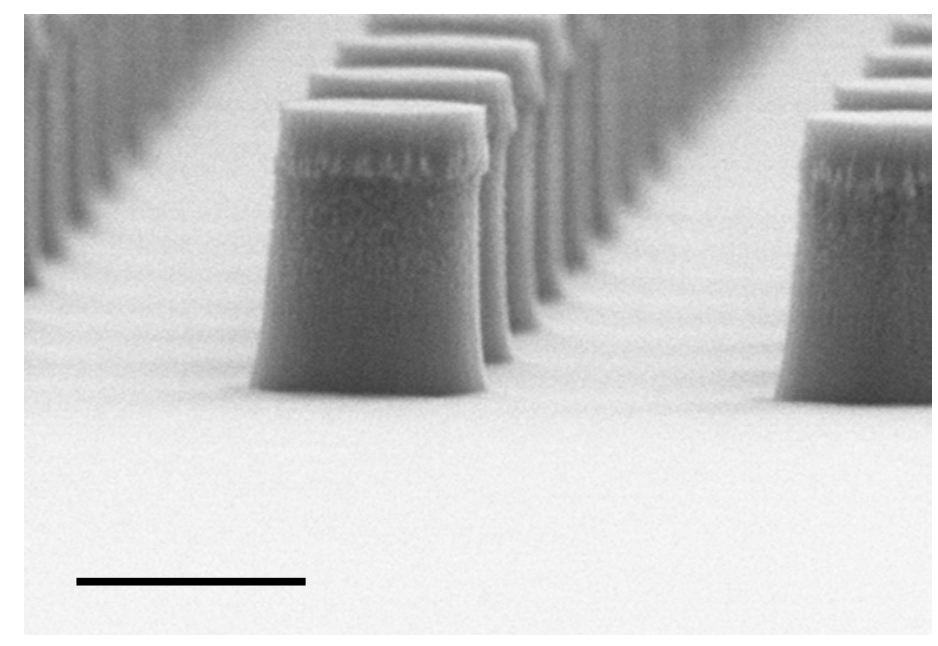

Figure 1. A scanning electron micrograph representing the types of patterned silicon substrates used in these studies. The scale bar is $1 \mu \mathrm{m}$.
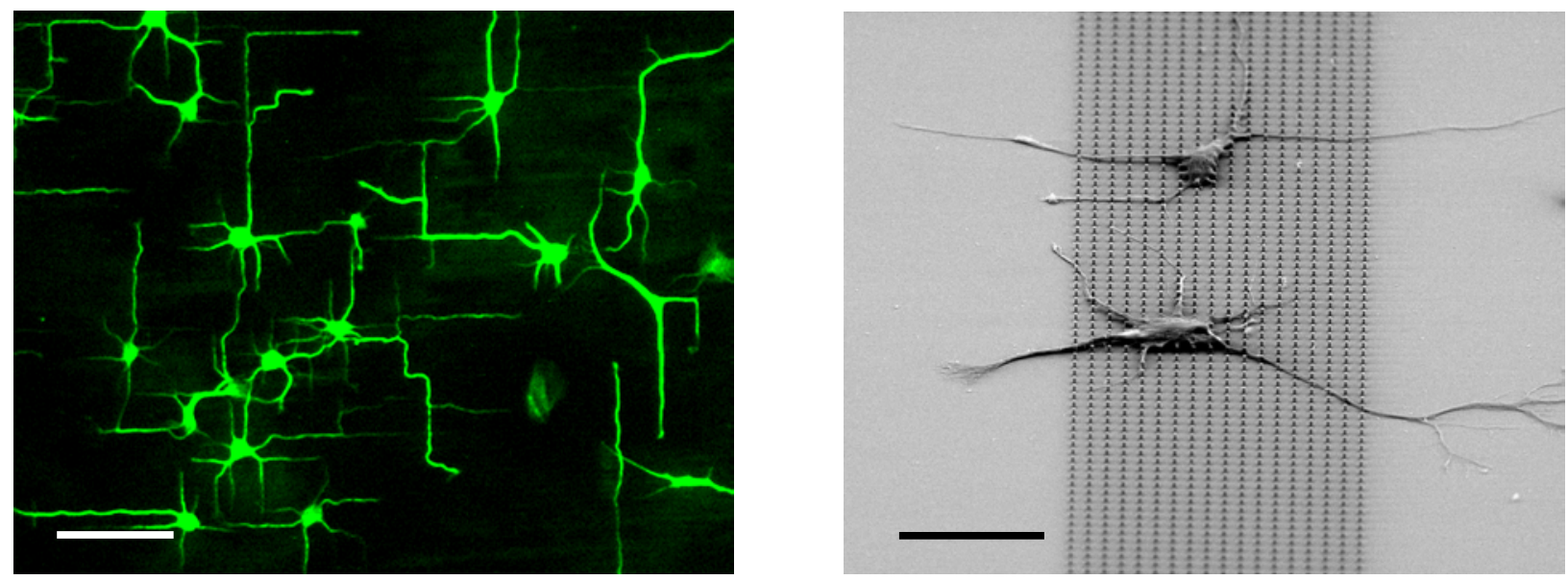

Figure 2. (A) A fluorescence micrograph of Alexa-488 stained hippocampal neurons fixed after 24 hours in culture on patterned silicon substrates. The scale bar is $50 \mu \mathrm{m}$. (B) A scanning electron micrograph showing process outgrowth in the regions of the pillars. The scale bar is $25 \mu \mathrm{m}$. 